\title{
Nonparabolicity and dielectric effects on addition energy spectra of spherical nanocrystals
}

\author{
J. Planelles ${ }^{\text {a) }}$ and M. Royo \\ Departament de Química Física i Analítica, UJI, Box 224, E-12080 Castelló, Spain \\ M. Pi \\ Departament ECM, Facultat de Física and $I N^{2}$ UB, Universitat de Barcelona, E-08028 Barcelona, Spain
}

(Received 9 May 2007; accepted 11 September 2007; published online 2 November 2007)

\begin{abstract}
An extension of the spin density functional theory simultaneously accounting for dielectric mismatch between neighboring materials and nonparabolicity corrections originating from interactions between conduction and valence bands is presented. This method is employed to calculate ground state and addition energy spectra of homogeneous and multishell spherical quantum dots. Our calculations reveal that corrections become especially relevant when they come into play simultaneously in strong regimes of spatial confinement. (C) 2007 American Institute of Physics. [DOI: 10.1063/1.2803722]
\end{abstract}

\section{INTRODUCTION}

The conduction band of semiconductors shows a nearly parabolic dispersion at the surroundings of the $\Gamma$ point and is commonly described by the one-band model, the interactions with the remaining bands being included in the electron effective mass. This is a sensible approach for wide-gap semiconductors. However, the small gap between the conduction and valence bands in narrow gap semiconductors has important nonparabolicity effects on the conduction band dispersion that warrant an improvement of the model. The use of an energy-dependent effective mass, as proposed by Kane, ${ }^{1,2}$ allows us to study narrow gap semiconductors still within the framework of the one-band model. Nevertheless, as the effective mass is self-consistently adjusted for each energy level, different kinetic energy, and therefore Hamiltonian operators, are employed to compute the different eigenvalues, so that the associate eigenfunctions are not necessarily orthogonal. In order to deal with orthogonal eigenfunctions, the electron effective mass is kept constant and a power series expanded dispersion relation is incorporated into the kinetic energy term of the Hamiltonian yielding a fourth (or higher) order differential Schrödinger-like equation. ${ }^{3}$ Although this approach has been successfully employed to study donor states in spherical quantum dots (QDs), ${ }^{4}$ it will be accurate only to the extent that the truncated power series succeed in describing the dispersion relation.

The energy-dependent effective mass, leading to nonorthogonal eigenfunctions, has been employed to study the electronic structure ${ }^{5}$ and optical excitations ${ }^{6,7}$ of quantum wells (QWs). Intersubband spin-density excitations in QWs have been addressed using the Kohn-Sham equations with energy-dependent effective mass. ${ }^{8}$ Extensive single particle studies of different shaped QDs, ${ }^{9-11}$ quantum rings, ${ }^{12}$ and artificial molecules ${ }^{13}$ have also been carried out. Recently, the energy-dependent effective mass approach has reproduced experimental effective masses obtained from optical

${ }^{\text {a)} E l e c t r o n i c ~ m a i l: ~ j o s e p . p l a n e l l e s @ q f a . u j i . e s ~}$ transition energies in $\mathrm{QWs}{ }^{14}$ and provided quantitative interpretations of capacitance voltage spectroscopy experiments. $^{15}$

Nonparabolicity has larger effects in the strong confinement regime and for excited states, where the energetic correction can even exceed the electron-electron interaction. ${ }^{9} \mathrm{~A}$ recent spin density functional theory ${ }^{16}$ (SDFT) study of a QD molecule with six electrons ${ }^{17}$ shows that the energydependent effective mass correction is comparable to the exchange energy that is obtained. Since the energy-dependent effective mass corrects the kinetic contribution to the total energy, minor effects coming from nonparabolicity could be expected in the weak confinement regime. In the present paper we incorporate nonparabolicity corrections into our SDFT code, ${ }^{18-20}$ which also accounts for polarization and self-polarization coming from dielectric mismatch between neighboring materials. We then explore the relevance of nonparabolicity corrections on the addition energies of homogeneous and multishell QDs subjected to different confinement regimes. We will show that the interplay of both dielectric mismatch and nonparabolicity effects may lead to relevant changes in the electronic structure and addition energies of QDs, the effect being particularly relevant in multishell QDs.

\section{THEORY AND COMPUTATIONAL DETAILS}

The formulation of the extension of the SDFT which accounts for position-dependent effective mass, polarization of the Coulomb interaction, and self-polarization coming from the dielectric mismatch between the QD and its surrounding medium (and also between neighboring materials in the case of multishell QDs) can be found in Ref. 18 and will not be outlined here for the sake of conciseness. For the present study we also implemented energy-dependent effective masses in a similar way to that reported in Ref. 17. It is worth pointing out that the use of orbital energy-depending masses is just an effective way of incorporating the perturbation produced by the valence bands on the conduction electron levels (the scheme we proposed here can be viewed 
as an approximation to the multiband calculation). Therefore, it is equivalent to leaving the kinetic energy operator as it is and introducing the appropriate perturbation external potential. As a consequence, same exchange-correlation functional should be employed in parabolic and nonparabolic calculations. In our implementation we basically include $m_{i \sigma}^{*}\left(E_{i \sigma}, \mathbf{r}\right)$ instead of $m^{*}(\mathbf{r})$ in the Kohn-Sham $(\mathrm{KS})$ equation, i.e., a single differential equation is replaced by a set of coupled differential equations

$$
\left[-\frac{\hbar^{2}}{2} \nabla \frac{1}{m_{i \sigma}^{*}\left(E_{i \sigma}, \mathbf{r}\right)} \nabla+V_{K S, \sigma}(n, \zeta)\right] \Phi_{i \sigma}(\mathbf{r})=E_{i \sigma} \Phi_{i \sigma}(\mathbf{r})
$$

that are solved up to simultaneous convergence. In the earlier equation $n$ is the total density and $\zeta$ represents the spin polarization. In terms of the spin-up and spin-down densities $n_{\sigma}(\mathbf{r})[$ where $\sigma=(+,-)$ labels the spin] they can be written as $n(\mathbf{r})=n_{+}(\mathbf{r})+n_{-}(\mathbf{r}) \quad$ and $\zeta(\mathbf{r})=\left[n_{+}(\mathbf{r})-n_{-}(\mathbf{r})\right] / n(\mathbf{r})$, respectively.

The energy-dependent effective mass in Eq. (1) is given by the Kane formula ${ }^{1,2}$

$$
\begin{aligned}
\frac{m_{0}}{m^{*}(E, \mathbf{r})}= & 1+\frac{2}{3} \mathcal{P}^{2}\left[\frac{2}{E+E_{g}(\mathbf{r})-V(\mathbf{r})}\right. \\
& \left.+\frac{1}{E+E_{g}(\mathbf{r})-V(\mathbf{r})+\Delta(\mathbf{r})}\right],
\end{aligned}
$$

where $m^{*}(E, \mathbf{r})$ is the energy- and position-dependent effective mass, $m_{0}$ is the free electron mass, $\mathcal{P}$ is the Kane parameter, and $E_{g}(\mathbf{r}), V(\mathbf{r}), \Delta(\mathbf{r})$ are the position-dependent band gap, confining potential, and spin-orbit parameter, respectively. For a practical implementation, we employ the relationship between the position- and energy-dependent effective mass $m^{*}(E, \mathbf{r})$ and the position-dependent effective mass at the bottom of the conduction band $m^{*}(0, \mathbf{r})$,

$$
\frac{\frac{m_{0}}{m^{*}(E, \mathbf{r})}-1}{\frac{m_{0}}{m^{*}(0, \mathbf{r})}-1}=\frac{\left[E+E_{g}(\mathbf{r})-V(\mathbf{r})+\frac{2}{3} \Delta(\mathbf{r})\right]\left[E_{g}(\mathbf{r})-V(\mathbf{r})\right]\left[E_{g}(\mathbf{r})-V(\mathbf{r})+\Delta(\mathbf{r})\right]}{\left[E_{g}(\mathbf{r})-V(\mathbf{r})+\frac{2}{3} \Delta(\mathbf{r})\right]\left[E+E_{g}(\mathbf{r})-V(\mathbf{r})\right]\left[E+E_{g}(\mathbf{r})-V(\mathbf{r})+\Delta(\mathbf{r})\right]} .
$$

Many implementations ${ }^{2,9-13,17}$ approximate the left-hand side of Eq. (3) by $m^{*}(0 . r) / m^{*}(E . r)$. Here we use Eq. (3) as it is.

In order to achieve a solution to the set of differential Eq. (1) we start by solving a single differential equation where $m_{i \sigma}^{*}\left(E_{i \sigma}, \mathbf{r}\right)$ is replaced by $m^{*}(0, \mathbf{r})$. From the $\Phi_{i \sigma}(\mathbf{r})$ orbitals and $E_{i \sigma}$ energies thus obtained we compute the total density $n$, spin polarization $\zeta$, and effective masses $m_{i \sigma}^{*}\left(E_{i \sigma}, \mathbf{r}\right)$ and build up the set of differential Eq. (1). In a second step, we solve these differential equations one by one to get the couple orbital and associate energy $\left[\Phi_{i \sigma}(\mathbf{r}), E_{i \sigma}\right]$ from the $i \sigma$ th differential equation. Then, from the new set of energies we get the next energy- and position-dependent effective masses $m_{i \sigma}^{*}\left(E_{i \sigma}, \mathbf{r}\right)$ and from the new orbitals the next total density $n$ and spin polarization $\zeta$ are obtained. The process is repeated up to simultaneous convergence of all differential equations. We can build the first order density matrix from a set of nonorthogonal orbitals or equivalently, we may first orthogonalize them and then construct the density matrix. We follow the second procedure in our code.

The good performance of our method computing addition energies of QDs with nearly parabolic conduction band has already been tested. ${ }^{18}$ Additionally, the use of energydependent effective masses has widely recognized as an appropriate effective way of incorporating the perturbation produced by the valence bands on the conduction energy levels (see the earlier section). Nevertheless, it is worth testing these approaches working together on a narrow-gap semiconductor QD. Certainly, the amount of experimental data on addition energies of spherical narrow-gap QDs in the pres- ence of relevant dielectric mismatch with the environment is rather scarce. We employ here experimental results by Banin et $a .^{21}$ on a $2.2 \mathrm{~nm}$ radius InAs QD embedded in an organic environment which first three experimental addition energies have already been very well reproduced by means of heavy atomistic pseudopotential calculations. ${ }^{22}$ In these calculations the dielectric constant of the organic environment was taken as a fitting parameter, yielding a value $\varepsilon_{\text {out }}=6$. In our test we have assumed, though, a value $\varepsilon_{\text {out }}=3$, which seems more reasonable for this kind of environment. ${ }^{23}$ The remaining parameters employed are those of bulk InAs, namely $m_{\mathrm{QD}}^{*}=0.023, \varepsilon_{Q D}=12.3, E_{g}^{\mathrm{QD}}=0.354 \mathrm{eV}, \Delta_{\mathrm{SO}}^{\mathrm{QD}}=0.41 \mathrm{eV}$, and for the external medium $m_{\text {out }}^{*}=1, \varepsilon_{\text {out }}=3, E_{g}^{\text {out }}=8 \mathrm{eV}, \Delta_{\mathrm{SO}}^{\text {out }}$ $=0 \mathrm{eV}$. Finally, the confining potential is assumed to be $V$ $=3.2 \mathrm{eV}$, the same as in Ref. 22. Parabolic/no-parabolic calculations predict (experimental data in parentheses) $\Delta_{1,2}$ $=0.22 / 0.19(0.14) \mathrm{eV}, \Delta_{2,3}=1.59 / 0.59(0.52) \mathrm{eV}$, and $\Delta_{3,4}$ $=0.20 / 0.14(0.14) \mathrm{eV}$. We see in this example that nonparabolic corrections work well improving all parabolic results, the improvement of $\Delta_{2,3}$ being especially relevant. The obtained results also show that our nonparabolic approach supplied with bulk parameters compares well with reported experimental data by Banin et al. ${ }^{21}$ Further improvement may be reached by fitting.

\section{RESULTS AND DISCUSSION}

We study the ground state and addition energies of spherical QDs built of narrow gap semiconductors versus the number $N$ of electrons. Spherical nanocrystals are commonly 
TABLE I. Parameters employed in this paper (taken from Ref. 25). Namely, effective mass $\left(m_{0}^{*}\right)$, dielectric constant $(\varepsilon)$, band gap $(E g)$, split of $(\Delta)$, and spatial confining potential barrier $(V)$.

\begin{tabular}{ccccc}
\hline \hline Material & $m_{0}^{*}$ & $\varepsilon$ & $\begin{array}{c}E g \\
(\mathrm{eV})\end{array}$ & $\begin{array}{c}\Delta \\
(\mathrm{eV})\end{array}$ \\
\hline $\mathrm{HgS}$ & 0.04 & 11.4 & 0.5 & 0.08 \\
$\mathrm{CdS}$ & 0.15 & 5.5 & 2.5 & 0.08 \\
$\mathrm{H}_{2} \mathrm{O}$ & 1 & 1.78 & 8. & 0.08 \\
& & $\mathrm{HgS} / \mathrm{H}_{2} \mathrm{O}$ & $\mathrm{CdS} / \mathrm{H}_{2} \mathrm{O}$ & $\mathrm{HgS} / \mathrm{Cds}$ \\
\hline$V(\mathrm{eV})$ & 4.15 & 2.8 & 1.35 \\
\hline \hline
\end{tabular}

synthesized in colloidal solutions. This technique allows the smallest QDs with sizes of very few nanometers to be produced. The QDs can be either homogeneous or onionlike, i.e., built up of concentric layers (shells) of different semiconductors with controllable shell thicknesses down to a single monolayer. In the present paper, we consider both homogeneous $\mathrm{HgS}$ nanocrystals and multishell double quantum well $\mathrm{CdS} / \mathrm{HgS} / \mathrm{CdS} / \mathrm{HgS} / \mathrm{CdS}$, similar to those experimentally synthesized in Ref. 24. Then, in all cases the surrounding medium of the QD is considered to be water. The parameters employed in our calculations for the different materials, namely effective masses, dielectric constants, band gaps, split-off parameters, and spatial confining potentials are the same as those employed in Ref. 25 and are shown in Table I. The bottom of the HgS conduction band is assumed to be the origin of energies in all cases. We have carried out calculations from 1 up to 25 electrons, using an orbital basis $1 s, 1 p$, $1 d, 1 f, 2 s$, and $2 p$.

\section{A. Homogeneous nanocrystal}

In this section we consider $\mathrm{HgS}$ QDs with 5 and $20 \mathrm{~nm}$ radii in water. From the $\mathrm{HgS}$ effective Bohr radius, $a_{0}^{*}$ $=a_{0} \varepsilon / \mathrm{m}^{*}=15 \mathrm{~nm}$, we see that the confinement regime of the small/large QD is strong/weak. For the time being the polarization coming from the dielectric mismatch is disregarded in our calculations, i.e., we use the dielectric constant of the QD for the whole system. We carry out three series of calculations versus the number $N$ of electrons in the QD ranging from 1 up to 25 . These are (i) independent particle (IP) calculations employing a parabolic effective mass; (ii) IP calculations with energy-dependent masses; and (iii) interacting electrons at the SDFT level with a parabolic effective mass (excluding dielectric mismatch effects). The results for the small and large QD, as representative of strong/weak confinement regime, are summarized in Figs. 1(a) and 1(b). Solid lines represent IP with parabolic mass, dashed lines correspond to IP including nonparabolicity corrections, and dotted lines refer to interacting particles with parabolic effective mass. The obtained results go along with expectations, i.e., nonparabolicity corrections increase with the number of electrons in the QD; in the strong confinement regime these corrections can be as large as the corrections originating from the electron-electron interaction [Fig. 1(a)] while in the weak confinement regime they are negligibly small [Fig. 1(b)]. The inset in Fig. 1(b) shows the evolution of the
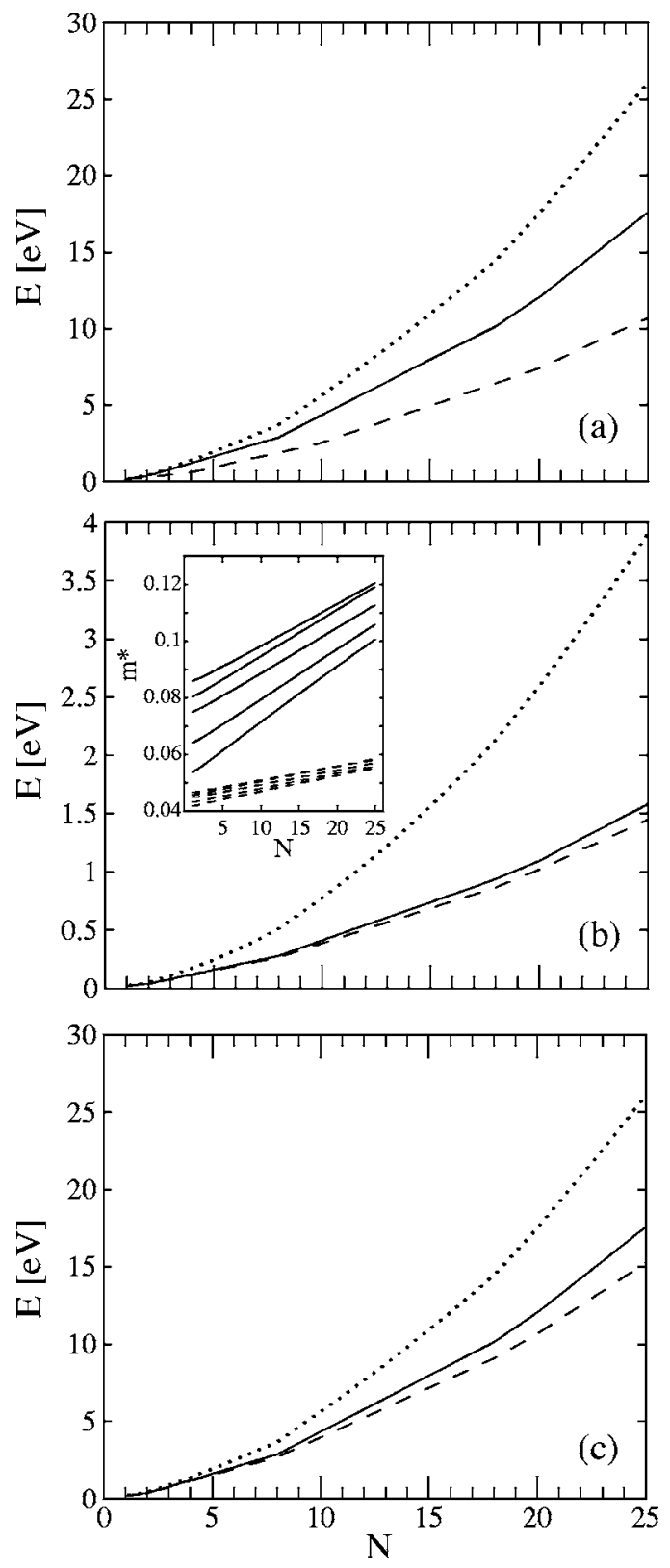

FIG. 1. Ground state energy vs number $N$ of electrons of a (a) $5 \mathrm{~nm}$ radius $\mathrm{HgS} \mathrm{QD}$, (b) $20 \mathrm{~nm}$ radius $\mathrm{HgS} \mathrm{QD}$, and (c) $5 \mathrm{~nm}$ radius QD defined by the $\mathrm{HgS}$ material parameters except for $E g$ that is set to $3 \mathrm{eV}$. Solid lines correspond to the parabolic IP calculations, dashed lines to IP including nonnonparabolicity corrections, and dotted lines to parabolic SDFT. The inset encloses the energy-dependent effective masses of the $5 \mathrm{~nm}$ radius $\mathrm{HgS}$ QD (solid lines) and $20 \mathrm{~nm}$ radius $\mathrm{HgS}$ QD (dotted lines) corresponding to the low-lying KS orbitals.

energy-dependent effective masses in the low-lying $1 s, 1 p$, $1 d, 1 f$, and $2 s$ states versus the number $N$ of electrons in the QD. Solid/dashed lines correspond to strong/weak confinement regimes. The results reveal that higher energy states have larger effective masses and that the electron effective mass increases almost linearly with $N$ (within the range studied here), these changes in effective masses being relevant only in the strong confinement regime. Interestingly, corrections coming from nonparabolicity and from electronelectron interactions are of opposite signs. In the strong confinement regime where nonparabolicity corrections are relevant [Fig. 1(a)], these corrections partially cancel out 

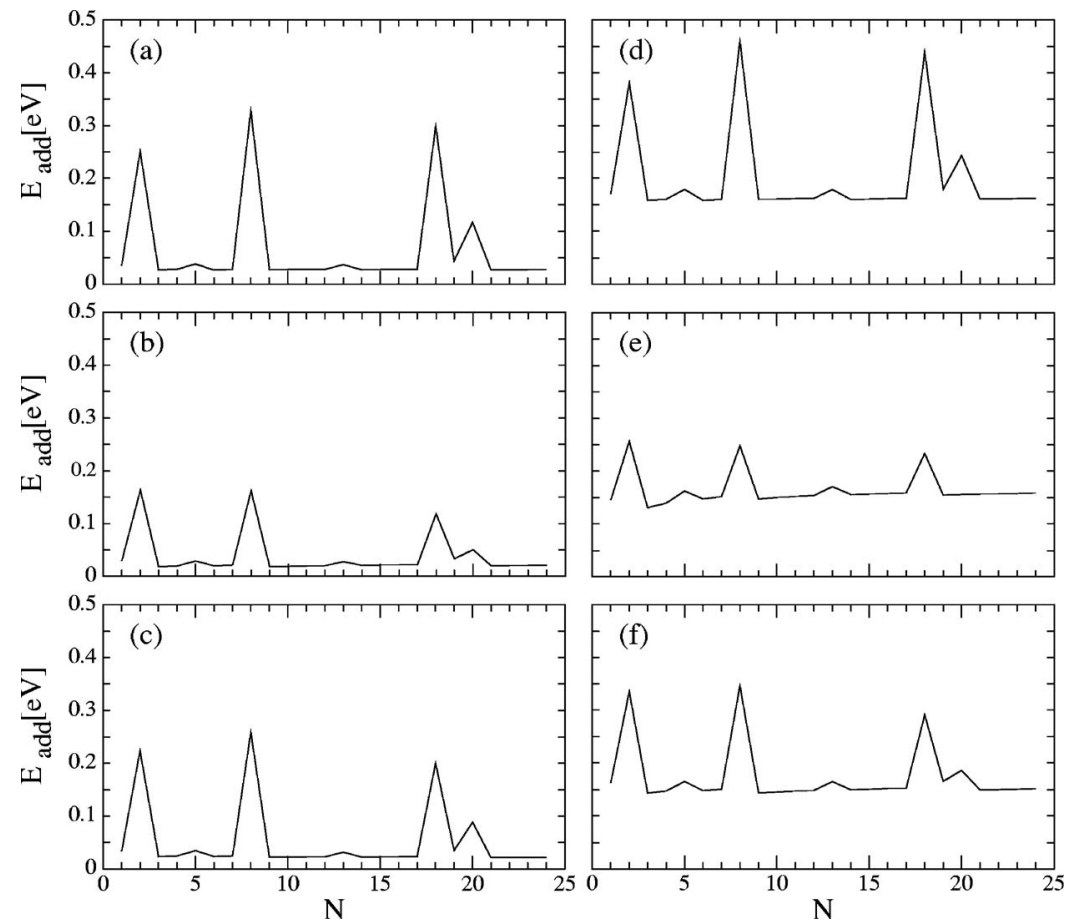

FIG. 2. Calculated addition energy spectra of a $5 \mathrm{~nm}$ radius $\mathrm{HgS}$ QD. (a) With a parabolic mass. (b) Including nonparabolicity corrections. (c) Same as (a) except that the QD material $E g$ is set to $3 \mathrm{eV}$. (d), (e), and (f) Same as (a), (b), and (c) but also including polarization coming from the dielectric mismatch between neighboring materials. with the electron-electron interaction corrections, so that calculations including both corrections yield energies close to those of the simple IP parabolic approach. In other words, it appears that simple parabolic IP calculations yield a reasonable, at least qualitative, description of the QD electronic structure in the strong confinement regime. In the weak confinement regime, where Coulomb interactions cannot be excluded for a reasonable description of the QD electronic structure, nonparabolicity corrections are negligibly small [see Fig. 1(b)], and it seems they can safely be ignored, at least for a qualitative description.

In order to show the extent to which these corrections can influence the electronic structure of QDs built of widegap semiconductor materials, we carried out a third series of calculations for a QD described by the same parameters as the small $\mathrm{HgS}$ nanocrystal except for the band gap that was set to $3 \mathrm{eV}$. Then, this QD is in the (same) strong confinement regime where nonparabolicity corrections become large. However, the results obtained reveal, as expected, that despite the strong confinement regime, nonparabolicity corrections are much smaller than those coming from Coulomb interactions [see Fig. 1(c)]. The behavior observed is therefore similar to narrow gap semiconductor QDs in the weak confinement regime [Fig. 1(b)].

So far we have excluded polarization coming from the dielectric mismatch and focused on total energies, the calculations revealing that nonparabolicity corrections are not small in the strong confinement regime, although no particular situations have been found in which these corrections play a fundamental role. Next, we consider a QD in the strong confinement regime, carry out calculations of the quite sensitive addition energy, ${ }^{26}$ and allow polarization effects to come into play. To this end, we study the small $5 \mathrm{~nm}$ radius $\mathrm{HgS}$ QD in water again and estimate addition energies including and excluding polarization effects coming from the dielectric mismatch between the QD and its surrounding me- dium (hereafter we will refer to these calculations as polarized and unpolarized) and with/without nonparabolicity corrections. The calculations are summarized in Fig. 2. Panels on the left- and right-hand side show unpolarized/polarized calculations. Top row panels correspond to parabolic effective mass while those in the middle row include corrections from nonparabolicity. As earlier, for the sake of comparison, we enclose a series of calculations on a wide-gap semiconductor QD (panels at the bottom of Fig. 2). Thus, the calculations shown in the panels in the middle and bottom rows correspond to QDs defined with the same parameters and geometry except for the band gap (set to 0.5 and $3.0 \mathrm{eV}$, respectively). The unpolarized calculations with parabolic effective mass, assumed to be the reference, are shown in Fig. 2(a). We see that polarization and nonparabolicity corrections in isolation do not yield qualitative changes in the addition energy profile [Figs. 2(b) and 2(d), respectively]. In all three panels (a), (b), and (d), one can neatly recognize that the shell filling is done according to the Afbau and Hund rules. Large peaks reveal complete shell filling, small peaks show half filling. Then, the observed filling sequence is $1 s^{2} 1 p^{6} 1 d^{10} 2 s^{2} 1 f^{x}$. Only when both corrections are simultaneously included, can a reconstruction be observed. The orbital $2 s$ is not filled before $1 f$ [see Fig. 2(e)]. The reconstruction disappears if the QD is built of a wide-band semiconductor material [Figs. 2(c) and 2(f)]. The earlier mentioned reconstruction originates from the following facts. On the one hand, polarization corrections increase the orbital energies, thus enhancing the nonparabolicity corrections. On the other hand, $s$-symmetry orbitals $(l=0)$ have a null centrifugal term $l(l+1) / m^{*} r^{2}$ while this term is relevant for $f$ orbitals $(l=3)$. When nonparabolicity corrections come into play and increase the effective masses, $1 f$ undergoes a larger stabilization than $2 s$, and it is filled first. This is not the case for the wide-gap semiconductor QD because it has small 

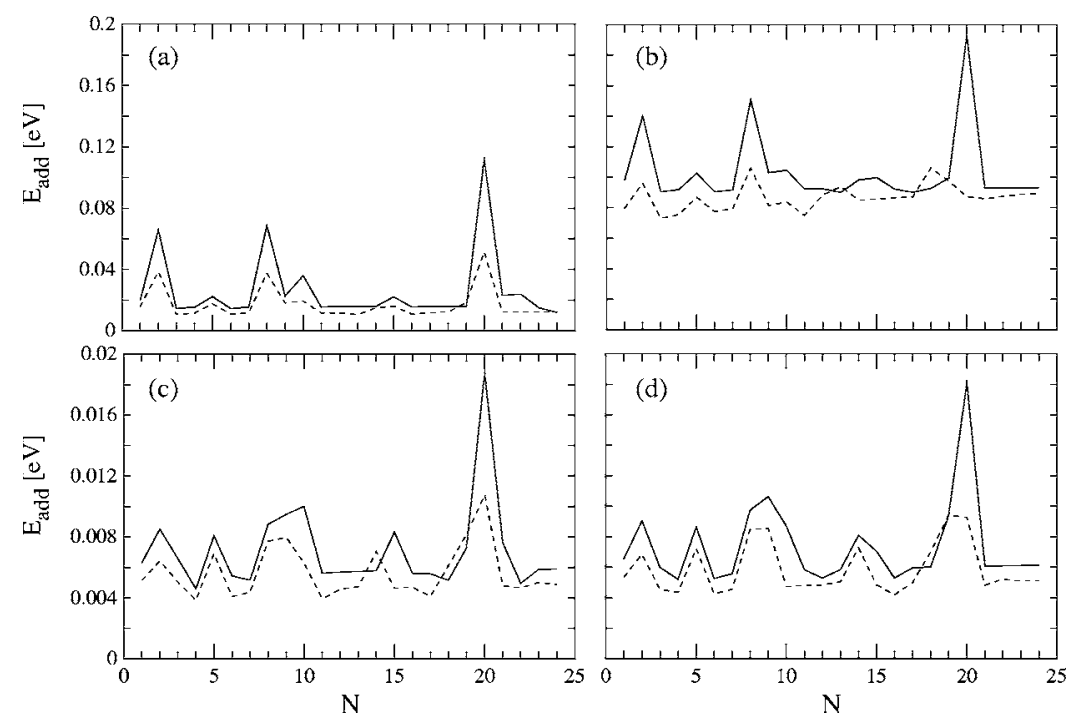

FIG. 3. Calculated SFDT addition energy spectra of a multishell $\mathrm{CdS} / \mathrm{HgS} / \mathrm{CdS} / \mathrm{HgS} / \mathrm{CdS}$ spherical QD double QW with core radius and shells of (a) $3 / 2 / 1 / 2 / 1 \mathrm{~nm}$, (b) same as (a) but also including polarization coming from the dielectric mismatch, (c) core radius and shells of 10/5/2/5/1 nm, and (d) Same as (c) but also including polarization. Solid lines represent calculations with parabolic effective mass. Dashed lines indicate that nonparabolicity corrections are included. nonparabolicity corrections, that never allow $1 f$ to reach an energy lower than that of $2 s$. Then $2 s$ is again filled first [see Fig. 2(f)].

In summary, in addition to exceptional situations where the interplay of nonparabolicity and polarization corrections may yield reconstructions and thus changes in the addition energy profile, the main effect that nonparabolicity corrections produce on the addition energy spectrum is a reduction in the height of the peaks [see e.g., Fig. 2(b) versus Fig. 2(a)] as the result of a higher degree of closeness of the orbital energies originating from an increase in the effective mass.

\section{B. Multishell quantum dots}

In the previous section we found a reconstruction in the addition energy spectrum originating from the relative closeness of orbital energies and the differential nonparabolicity effects on energy levels with distinct angular momentum quantum number $l$. Double quantum wells are good candidates for a nonregular energy spectrum. Thus, in this section we consider a multishell $\mathrm{CdS} / \mathrm{HgS} / \mathrm{CdS} / \mathrm{HgS} / \mathrm{CdS}$ spherical QD double QW like those synthesized in Ref. 24. The parameters employed in our calculations can be found in Table I. It is no straightforward task to unambiguously define the confinement regime of these heterogeneous systems in which the electronic density is mainly concentrated in the wells, but it seems reasonable to assume that the volume of the wells will basically determine it.

We consider two systems with different confinement strengths defined by the following core radius and shell thickness, $3 / 2 / 1 / 2 / 1$ and 10/5/2/5/1 nm, respectively, and carry out polarized/unpolarized calculations with/ without the inclusion of nonparabolicity corrections. The results can be seen in Fig. 3. Panels (a) and (b) in Fig. 3 correspond to the stronger spatial confinement while panels (c) and (d) correspond to the weaker confinement. Solid/ dashed lines refer to parabolic/nonparabolic effective masses.

First we analyze the smaller (strong confined) system. The reference addition energy spectrum [unpolarized and parabolic, solid line in Fig. 3(a)] shows a regular sequential filling up to 20 electrons $\left(1 s^{2} 1 p^{6} 2 s^{2} 1 d^{10}\right)$. Then, a shoulder emerges at $N=22$ involving the $2 p$ orbital. This shoulder disappears when nonparabolicity corrections are included (dashed line in the same panel), which reveals a regular filling of the $1 f$ orbital, due to the fact that $1 f(l=3)$ undergoes a larger energy stabilization coming from nonparabolicity corrections than $2 p(l=1)$, this fact being mainly related to the centrifugal term. Except for this reconstruction, unpolarized calculations with and without nonparabolicity corrections yield similar addition spectra. The main difference is a reduction in the height of the peaks when corrections are taken into account. This reduction comes from a higher degree of closeness of the orbital energies as the effective masses increase, due to nonparabolicity effects.

When polarization is taken into account in the parabolic mass calculation [solid line in Fig. 3(b)] no relevant changes in the addition energy spectrum can be seen. We may mention the disappearance of the shoulder at $N=22$ and some "noise" when filling orbital $1 d$. However, when both polarization and nonparabolicity corrections are simultaneously taken into account [dashed line in Fig. 3(b)] severe changes in the addition energy spectrum occur. On the one hand, fulland half-filling peaks become similar in height. On the other hand, the largest peak at $N=20$ corresponding to the full filling of orbital $1 d$ drops out.

The shell structure of the larger multishell QD is not neatly apparent in the addition spectrum. However, the reference unpolarized and parabolic addition energy spectrum [solid line in Fig. 3(c)] still shows peaks at $N=2\left(1 s^{2}\right), N$ $=5\left(1 s^{2} 1 p^{3}\right), \quad N=8\left(1 s^{2} 1 p^{6}\right), \quad N=10\left(1 s^{2} 1 p^{6} 2 s^{2}\right), \quad N$ $=15\left(1 s^{2} 1 p^{6} 2 s^{2} 1 d^{5}\right)$, and $N=20\left(1 s^{2} 1 p^{6} 2 s^{2} 1 d^{10}\right)$, although the profile is far from being as regular as the one for the smaller multishell QD [solid line in Fig. 3(a)]. When nonparabolicity corrections are taken into account [dashed line in Fig. 3(c)] the most significant change observed is a peak flip from $N$ $=15$ (corresponding to the filling $1 s^{2} 1 p^{6} 2 s^{2} 1 d^{5}$ ) up to $N$ $=14$ (when corrections are included and that corresponds to $\left.1 s^{2} 1 p^{6} 2 s^{2} 1 d^{5}\right)$. Additionally, as in all previously studied examples, the height of the peaks are reduced due to a higher 
degree of closeness of orbital energies coming from an increase in effective mass induced by nonparabolicity.

Accounting for polarization while employing a parabolic mass does not introduce qualitative changes in the addition energy spectrum [solid line in Fig. 3(d)] except, as earlier, for the shift from $N=15$ to $N=14$ of the half filling of $1 d$. Finally, in contrast with the results corresponding to the smaller (more strongly confined) multishell QD, by including both polarization and nonparabolicity corrections, no severe changes occur in the spectrum [dashed line in Fig. 3(d)]. This is related to the fact that both corrections are smaller in larger systems.

\section{CONCLUSIONS}

In this paper we have studied the relevance of corrections coming from nonparabolicity on the ground state and addition energies of homogeneous and multishell spherical QDs and their interplay with effects coming from the presence of dielectric mismatch. To this end we employ a SDFT approach modified to include polarization coming from the dielectric mismatch between neighboring materials and nonparabolicity originating from the interaction between conduction and valence bands. This approach basically translates into a set of coupled differential equations that are solved up to simultaneous convergence.

Our calculations show that homogeneous QDs built of narrow gap semiconductor materials show large nonparabolicity energy corrections if the QD regime of spatial confinement is strong. In such a case, these corrections are of the same order and opposite sign as corrections coming from electron-electron interaction and partially cancel each other out, so that the simple parabolic IP approach yields reasonable, at least qualitative, results. A similar conclusion applies to the addition energy spectra. Since most of the spherical QDs are chemically synthesized in colloidal solutions, in our calculations we have incorporated the polarization originated by the dielectric mismatch between the QD and its surroundings that we consider to be water (and also between different materials in the case of multishell QDs). Our results reveal that the interplay of polarization and nonparabolicity corrections may yield reconstructions in the addition energy spectrum. Our analysis points out two reasons for this. On the one hand, polarization increases orbital energies thus enhancing nonparabolicity corrections. On the other hand, the different centrifugal terms felt by orbitals with different angular momentum quantum number $l$ include the effective mass in the denominator. As a result, nonparabolicity corrections may even flip the orbital energy and the filling sequence of orbital with different $l$. As a major conclusion we can say that the calculations on addition energy spectra of multishell
QDs is the scenario where corrections play a prominent role. The most significant changes arise in the stronger spatial confinement when the dielectric mismatch effects are also relevant and come into play together with nonparabolicity corrections.

\section{ACKNOWLEDGMENTS}

The authors thank M. Barranco for useful discussions. Financial support from Spanish MEC-DGI Project Nos. CTQ2004-02315/BQU and FIS2005-01414, UJI-Bancaixa Project No. P1-1B2006-03, and Generalitat de Catalunya Grant No.2005SGR00343 is gratefully acknowledged. UJIBancaixa FPI (M.R.) grant is also acknowledged.

${ }^{1}$ E. O. Kane, J. Phys. Chem. Solids 1, 249 (1957).

${ }^{2}$ G. Bastard, Wave Mechanics Applied to Semiconductor Heterostructures (Les Editions de Physique, Les Ulis Cedex, 1988).

${ }^{3}$ A. Persson and R. M. Cohen, Phys. Rev. B 38, 5568 (1988).

${ }^{4}$ C. Bose, K. Midya, and M. K. Bose, Physica E (Amsterdam) 33, 116 (2006).

${ }^{5}$ W. Chen and T. G. Andersson, Phys. Rev. B 44, 9068 (1991).

${ }^{6}$ D. Huang, Phys. Rev. B 53, 13645 (1996).

${ }^{7}$ X. Chen, Physica B (Amsterdam) 270, 88 (1999).

${ }^{8}$ C. A. Ullrich and M. E. Flatté, Phys. Rev. B 66, 205305 (2002).

${ }^{9}$ Y. Li, O. Voskoboynikov, C. P. Lee, and S. M. Sze, Solid State Commun. 120, 79 (2001).

${ }^{10}$ Y. Li, J. L. Liu, O. Voskoboynikov, C. P. Lee, and S. M. Sze, Comput. Phys. Commun. 140, 399 (2001).

${ }^{11}$ Y. Li, O. Voskoboynikov, C. P. Lee, and S. M. Sze, Comput. Phys. Commun. 141, 66 (2001).

${ }^{12}$ O. Voskoboynikov, Y. Li, H. M. Lu, C. F. Shih, and C. P. Lee, Phys. Rev. B 66, 155306 (2002).

${ }^{13}$ Y. Li, O. Voskoboynikov, C. P. Lee, and S. M. Sze, Comput. Phys. Commun. 147, 209 (2002).

${ }^{14}$ N. Kotera and K. Tanaka, Physica E (Amsterdam) 32, 199 (2006).

${ }^{15}$ I. Filikhin, E. Deyneka, and B. Vlahovic, Physica E (Amsterdam) 31, 99 (2006); I. Filikhin, V. M. Suslov, and B. Vlahovic, Phys. Rev. B 73, 205332 (2006).

${ }^{16}$ U. von Barth and L. Hedin, J. Phys. C 5, 1629 (1972).

${ }^{17}$ J. L. Liu, J. H. Chen, and O. Voskoboynikov, Comput. Phys. Commun. 175, 575 (2006).

${ }^{18}$ M. Pi, M. Royo, and J. Planelles, J. Appl. Phys. 100, 073712 (2006)

${ }^{19}$ M. Royo, J. Planelles, and M. Pi, Phys. Rev. B 75, 033302 (2007).

${ }^{20}$ W. Kohn and L. J. Sham, Phys. Rev. 140, A1133 (1965).

${ }^{21}$ U. Banin, Y. Cao, D. Katx, and D. Millo, Nature (London) 400, 542 (1999).

${ }^{22}$ A. Franceschetti, A. Williamson, and A. Zunger, J. Phys. Chem. B 104, 3398 (2000).

${ }^{23}$ See, e.g., M. Drndić, M. V. Jarosz, N. Y. Morgan, M. A. Kastner, and M. G. Bawendi, J. Appl. Phys. 92, 7498 (2002).

${ }^{24}$ M. Braun, C. Burda, and M. A. El-Sayed, J. Phys. Chem. A 105, 5548 (2001).

${ }^{25}$ K. Chang and J. B. Xia, Phys. Rev. B 57, 9780 (1998); W. Jaskólski and G. W. Bryant, ibid. 57, R4237 (1998); A. D. Yoffe, Adv. Phys. 50, 1 (2001).

${ }^{26}$ Addition energies are defined as $E_{\text {add }}(N)=E(N+1)+E(N-1)-2 E(N)$, where $E(N)$ is the total energy of the QD populated with $N$ electrons. Experimentally, the shell structure of QDs is often inferred from their addition spectrum that can be obtained from, e.g., capacitance voltage spectroscopy experiments. 